\title{
IMPACTO AMBIENTAL Y SOCIOECONÓMICO DE LAS ACTIVIDADES ACUÍCOLAS Y PESQUERAS EN LA REGIÓN TACNA
}

Responsable: Blgo. Pesq. Nelver Coronel Flores

\begin{abstract}
RESUMEN
En el presente trabajo se detallan, en primer lugar, las caracteristicas medioambientales de la región Tacna, desde el punto de vista bioecológico, haciendo énfasis en aquellas que tienen importancia o relación con las actividades acuicolas y pesqueras que se desarrollan en la región. En segundo lugar, se analiza cómo y en qué medida estas actividades vienen ejerciendo algún tipo de impacto, tanto en el medio ambiente como también sobre la socioeconomía de los asentamiento humanos localizados en su entorno, llegándose a determinar que estas actividades mayormente no están todavia afectando negativamente sobre el medio ambiente, pero si positivamente sobre la socioeconomía, sobre todo de los pobladores de las zonas altoandinas, en quienes se observa un mejoramiento de su calidad de vida, por un incremento adicional a sus ingresos económicos por esta nueva actividad productiva del cultivo de trucha arco iris en rios y lagunas de la región Tacna.

\section{ABSTRACT}

The present work "Environmental and Socioeconomic Impact of Acquacultural and Fishing Activities on the Region of Tacna" describes first the environmental characteristics of the region from the bioecological angle, emphasizing on those activities important to acqualcultural and fishing activities. Also, are analyzed how and to what degree these activities have had an impact on the environment and on the socioeconomic conditions of the populations in the area of influence. The results show that they have not yet had a negative effect on the environment, but a positive impact on the socioeconomic status of the communities living in the highlands for whom an improvement in their quality of life was observed. They have experienced an increase in their income due to the productive activity of rainbow trout culture in the rivers and lakes of Tacna region.
\end{abstract}

\section{INTRODUCCIÓN}

En el ámbito de la región Tacna, se vienen desarrollando diversas actividades dentro de los campos de la extracción, cultivos y procesamiento de recursos hidrobiológicos, tanto marinos como continentales. Estas actividades generan una parte importante del total del producto bruto interno de la región y sustentan la economía de un buen sector de la población.

Se dispone de información estadística referente a montos de extracción y procesamiento de recursos hidrobiológicos, la cual es consolidada por la Dirección Regional de la Producción de Tacna, pero hasta la fecha no se han realizado mayormente estudios referente a los probables impactos que estas actividades acuicolas y pesqueras estarian causando sobre los recursos, su entorno y sobre la socioeconomía de las poblaciones urbana y rural de Tacna.

Es innegable que la extracción, cultivo y procesamiento de recursos hidrobiológicos, si es que no se realizan de una manera racional, controlada y sustentable, causan efectos perjudiciales, primero sobre los mismos recursos y luego sobre otras actividades que se desarrollan en su entorno, produciendo muchas de las veces un deterioro del ambiente, en desmedro de la economía de gran parte de la población que depende de estas acitividades productivas.

\section{CARACTERIZACIÓN AMBIENTAL $Y$ SOCIOECONÓMICA DE LA REGIÓN TACNA}

\subsection{Ubicación Geográfica y Demografía}

El departamento de Tacna, ahora región Tacna, se encuentra ubicado en el extremo sur occidental del Perú, enmarcado por las coordenadas geográficas $16^{\circ} 58^{\prime}$ y $18^{\circ} 21^{\prime} 34,8^{\prime \prime}$ LS y $69^{\circ} 28^{\prime}$ y $71^{\circ} 10^{\prime} \mathrm{LW}$; en altitudes que van de los 0,0 a los $5000 \mathrm{msnm}$. Políticamente, está conformado por 04 provincias y 27 distritos. La población total de la región Tacna para el año 2002 era de 294214 habitantes aproximadamente y la densidad poblacional de 18,31 habitantes $/ \mathrm{km} 2$, donde el $89,4 \%$ corresponde a la provincia de Tacna (263 047 hbtes.), 4,3\% a Jorge Basadre (12 561 hbtes.), $3,4 \%$ a Candarave ( 9936 hbtes.) y $2,9 \%$ a Tarata (8670 hbtes.).

\subsection{Areas Geográficas}

En la región Tacna, se distinguen tres áreas o zonas claramente delimitadas:

a) Marítima.- Tiene un área de $44448 \mathrm{~km}^{2}$ que abarca la zona del litoral, desde el límite con Chile (Línea de la Concordia) hasta el límite con la región Moquegua en $120 \mathrm{~km}$ y desde la costa hasta las 200 millas mar adentro. 
b) Costa.- Que ocupa $7767,7 \mathrm{~km}^{2}(48,3 \%$ de la superficie total departamental). Está comprendida desde 0 hasta los $2000 \mathrm{msnm}$, se caracteriza por ser desértica y húmeda, con extensas pampas de amplitud agrícola, donde se identifican tres valles importantes: Caplina, Sama y Locumba; sobresaliendo en el valle del Caplina las irrigaciones de "La Yarada" y "Los Palos", con $6500 \mathrm{~h}$. de cultivo que son irrigadas con agua subterránea.

c) Andina.- Ocupa un área de $8308 \mathrm{~km}^{2}$ de la superficie departamental (lo que representa el $51,7 \%$ ). Abarca desde los 2000 hasta más de 5 $000 \mathrm{msnm}$. Se caracteriza por presentar 2 zonas: la Interandina (desde los 2800 hasta los 4000 msnm.), conformada por valles intermedios, y la Alto andina (comprendida desde los 4000 hasta más de $5000 \mathrm{msnm}$ ) dedicada básicamente a la crianza de camélidos sudamericanos y en menor proporción a los ovinos a través del pastoreo en bofedales. Es una zona muy accidentada por la presencia de la Cordillera Occidental Volcánica, que le confiere condiciones totalmente diferentes a las de la Costa.

\subsection{Dinámica Económica de la Zona}

Las actividades que más aportaron al Producto Bruto Interno (PBI) Departamental en el 2001 fueron las actividades de servicios y la minería debido a la explotación de cobre de Toquepala, siendo el citado producto el de mayor exportación no sólo de la región sino del país. Las actividades que siguen en importancia en la economia tacneña son: el sector Industrial en 7,6\% Construcción en 7,2\% y el Agropecuario en $7,1 \%$.

\section{RECURSOS HIDRICOS E HIDROBIOLÓGICOS}

\subsection{Cuencas y Disponibilidad de Agua}

Se estructuran básicamente en la región Tacna cuatro cuencas hidrográficas: Locumba, Sama, Caplina-Uchusuma y Maure.

a) Cuenca Locumba.-El río Locumba se forma de la unión de los ríos Curibaya e llabaya. Está intimamente ligado a la explotación de la laguna de Aricota, irrigando los valles de Locumba e Ite.

b) Cuenca Sama.- El río Sama debe su origen a los ríos Jarumas y Quequesani. Sus excedentes son del orden de los $40 \mathrm{~m}^{3}$ anuales en la época de verano (enero a marzo) debido a las precipitaciones de las partes altas, exhibiendo en contraparte constantes déficit en los restantes períodos del año.

c) Cuenca Caplina-Uchusuma.- Básicamente estructurada sobre el cauce del río Caplina, regando el denominado "Valle Viejo" de Tacna. Se considera dentro de este sistema al Canal Uchusuma, que recoge agua del río del mismo nombre y quebradas de Vila Vilani. Son sus aguas las que abastecen a la ciudad y el valle de Tacna, exhibiendo en razón a ello un desbalance constante entre su caudal y los requerimientos existentes en la zona.

d) Cuenca Maure.- Sus aguas se originan en la Laguna de Vilacota y su recorrido es de NO a SE y llegan hacia el vecino pais de Chile. Sus descargas medias mensuales fluctúan entre 1 $363 \mathrm{~m}^{3} / \mathrm{s}$ y una máxima de $9963 \mathrm{~m}^{3} / \mathrm{s}$. Exhibe, en contraparte, elevadas concentraciones minerales, producto del geotermalismo prevaleciente en lazona.

Otros recursos superficiales importantes de la región son indudablemente las lagunas y represas localizadas en la zona andina de Tacna, las que contribuyen adicionalmente en la generación de servicios (energía eléctrica) y el soporte para el aparato productivo en un ámbito suprarregional.

En general, la Región de Tacna presenta una elevada restricción de agua que reduce el techo productivo agropecuario, agroindustrial, minero, pesquero, de consumo humano y de demanda turística

\subsection{Recursos Hidrobiológicos:}

a) En La Zona Litoral.- Esta zona viene a ser la ribera del mar, que se extiende desde la Línea de La Concordia en el sur, pasando sobre la superficie de la Fosa Tectónica donde se establecen las irrigaciones de Los Palos y La Yarada, continúa hacia el norte de la desembocadura del rio Sama, por las playas rocosas de la Boca del Río, Los Hornos y Vila Vila; sigue por los acantilados de la cadena costanera hasta Punta Huaca Luna en el límite con Moquegua. En esta zona se encuentran dos tipos de costas: arenosas y rocosas.

- Costas Arenosas.- Se caracterizan porque en ellas se encuentran especies como: "macha" (Mesodesma donacium), "muy muy" (Emerita analoga), "jaiva" (Cancer polyodon), "cangrejo ermitaño" (Pagurus $s p$ ) y "cangrejo carretero". También se tienen otras especies pequeñas que podrian ser consideradas componentes de la fauna ecológica de este tipo de biocenosis y la presencia de peces pleuronéctidos como el "lenguado" (Paralichtyes adspersus), entre otras. Así mismo, el espacio de la zona, sometido a la alternancia de mareas y el poco espesor de la capa liquida, atraen hacia las regiones litorales de arena a una gran variedad de aves muy relacionadas con la fauna marina por sus costumbres y alimentación.

- Costas Rocosas.- Constituyen el lugar predilecto para los moluscos nadadores, debido a que en ellas encuentran fácilmente 
su alimento y refugio; entre ellos se tiene a los "pulpos" (Octopus sp), los equinodermos como la "estrella de mar" (Luidia bellonae), "erizo de mar" (Echinus sculenta) "guamaria", etc., que encuentran allí un medio favorable para el crecimiento, existiendo en mayor variedad y cantidad que en los fondos de arena. Numerosos peces prefieren también los lugares rocosos por su agua con excelente concentración de oxígeno (frecuentes olas). Además, constituyen el hábitat favorito de especies de moluscos univalvos "chanque" (Concholepas concholepas), "lapas" (Fisurella $s p$ ), "caracol" (Thais chocolata) y Tegula sp, "Choro" (Aulacomya ater), el "cangrejo azul", el "camaroncillo marino" y la "nutria común".

b) En la Zona Continental.- Como se indicó, los recursos hidricos continentales en la Región Tacna, están distribuidos en cuatro cuencas principales: Locumba, Sama, CaplinaUchusuma y Maure. Dentro de estas cuencas hídricas existen recursos lóticos (ríos, riachuelos y manantiales) y lénticos (lagunas y represas), con posibilidades de explotación piscícola en forma natural y artificial. Las principales especies que habitan estas aguas continentales son la trucha arco iris (Oncorhynchus mykiss), la trucha de arroyo (Salvelinus fontinalis) y el camarón de rio (Cryphiops caementarius).

\section{SITUACIÓN DEL SECTOR PESQUERO REGIONAL}

La región Tacna presenta una zona litoral y plataforma o zócalo continental con abundantes recursos marinos (existen condiciones óptimas para la vida acuática), con más de 342 especies con alto valor económico, que hacen de Tacna una región con un desarrollo pesquero creciente; y es que, al margen de su poco significativo (aún) impacto en la producción regional, la pesquería exhibe, como pocos sectores, un nivel de crecimiento sostenido e importante durante la última década, el mismo que implica adicionalmente una mayor absorción de mano de obra.

Podemos resumir en breve la problemática del subsector pesquero, en torno a lo siguiente:

- Limitado conocimiento técnico-científico de los recursos hidrobiológicos.

- Depredación de bancos naturales, por la sobreexplotación.

- Escasa tecnificación y modernización de la flota pesquera.

- Falta de promoción en la fase de procesamiento para que se adecúe a las nuevas tecnologías.

- Ausencia de centros de mercadeo que permitan el ordenamiento de la comercialización, asi como en los aspectos de higiene y sanidad.

-.Escasos recursos hídricos que limitan el desarrollo de la acuicultura continental.

- Patrones de consumo rigidos orientados a especies tradicionales, lo que origina un bajo consumo per cápita de los productos pesqueros.

- Planes de ordenamiento no adecuados a la realidad regional.

- Falta de conciencia del pescador artesanal, respecto a la pesca responsable.

- Insuficiente tecnologia y presupuesto para desarrollar la acuicultura.

- Insuficiente presupuesto institucional.

\subsection{Recursos Humanos:}

El pescador artesanal marítimo es un trabajador "sui géneris", que realiza sus labores o faenas pesqueras con predominio del trabajo manual, sin emplear embarcaciones pesqueras (pescador de ribera) o empleando embarcaciones de hasta 32,6 ton. de capacidad de bodega y $15 \mathrm{~m}$ de eslora, utilizando diferentes artes y aparejos de pesca para capturar especies destinadas al consumo humano directo al estado fresco $\mathrm{y} / \mathrm{o}$ refrigerado, congelado, enlatado y curado.

Los pescadores artesanales de los desembarcaderos de Vila Vila y Puerto Pesquero Morro Sama y áreas de pesca como Boca del Río, Llostay, Santa Rosa, Kulauta. Los Palos, Punta Picata, etc. suman alrededor de 700 pescadores; también se tiene aproximadamente un número de 200 pescadores no embarcados dedicados a labores de estiba, limpieza, etc.

En la zona de Santa Rosa, Kulauta. Los Palos, La Yarada, Llostay y Boca del Río, se tiene a los extractores de orilla, quienes están dedicados exclusivamente a la extracción de peces (Iorna, lenguado, corvinilla, etc.) que son comercializados directamente en los diferentes mercados de la ciudad de Tacna.

Se debe indicar que, desde el año 1998, por acción del fenómeno "El Niño", el recurso "macha" ha desaparecido, por tal motivo los extractores de este bivalvo en su mayoria han retornado a su lugar de origen, mientras que otros se dedican a la pesca con cortina y/o a la agricultura de pan llevar.

Alos agentes que participan directamente en las actividades pesqueras artesanales se los clasifica en la siguientes categorías:

- Pescadores Artesanales: Pinteros, rederos, pulmoneros de ribera, buzos civiles con patente, recolectores de machas, extractores de mariscos, recolectores de algas marinas, recolectores de camarón de río.

- Armadores Artesanales: Propietarios de embarcaciones pesqueras de 0,5 a 30 Ton. de capacidad de bodega.

- Procesadores Artesanales: Aquellos que se 
dedican al procesamiento primario o curado de los productos hidrobiológicos para consumo humano directo (CHD).

El número de personas dedicadas a la actividad extractiva de recursos hidrobiológicos en la Región Tacna según área es el siguiente:

- Pescador artesanal área marina:700

Personas.

- Pescador artesanal área continental :200

- Pescador artesanal (servicios):50

Los pescadores artesanales marítimos se encuentran organizados en las siguientes Asociaciones y/o Sindicatos:

- Asociación de Pescadores Artesanales de Puerto Pesquero Morro Sama Tacna."

- Asociación de Pequeños Propietarios de Embarcaciones Artesanales de Morro SamaVila Vila.

- Asociación de Armadores de PescaArtesanal para Consumo Humano Directo del Puerto Pesquero de Morro Sama.

- Asociación del Sindicato de Pescadores Artesanales de Vila Vila y Anexos.

- Asociación de Pescadores Artesanales de la Boca delRío.

- Asociación de Pescadores Artesanales y de Buzos Civiles Punta Grau.

- Asociación de Pescadores Artesanales y Extractores de Productos Hidrobiológicos Playa Santa Rosa.

- Asociación de Extractores de Productos Hidrobiológicos "ElPacífico".

- Asociación de Pescadores Artesanales "Agro San Pedro" Los Palos.

- Asociación de Buzos Civiles y Pescadores Artesanales de la Heroica Ciudad de Tacna.

- Asociación de Pescadores Artesanales Cruz de Picata Ite Tacna.

- Asociación de Pescadores Artesanales Virgen de Chapi Los Palos.

Por ser la pesqueria una actividad bastante aleatoria, los ingresos de los pescadores artesanales solamente les alcanza para subsistir; esta situación es causada por la imposición de bajos precios por parte de los intermediarios, lo que conduce, en el caso de los armadores o pescadores propietarios o copropietarios a que no dispongan de recursos económicos para mantener, reparar y modernizar sus embarcaciones y, en el caso de los asalariados, a sólo trabajar por temporadas de pesca, pues por el bajo salario se ven obligados a dedicarse a otras actividades.

En cuanto a la pesca continental, tenemos que en el caso específico de las comunidades altoandinas de Huaytire y Mamaraya, ubicadas en las provincias de Candarave y Tarata, respectivamente, el reparto de utilidades corresponde a todos los socios, de acuerdo a la extracción y precio de venta del producto de cosecha que es la trucha arco iris.
Los pescadores artesanales de la actividad extractiva continental, se agrupan en las siguientes organizaciones:

- Asociación de Pescadores Artesanales de la Laguna Suches-Huaytire.

- Asociación de Extractores de Trucha de la Represa Jarumas-Anexo Mamaraya.

- Asociación de Pesquería Artesanal Santa Magdalena-Susapaya

- Asociación de Pescadores Artesanales de Trucha Quilahuani

- Asociación de Extractores y Criadores Artesanales de Camarón José Olaya Balandra.

- Asociación Hidrobiológica Sama Baja.

\subsection{Comercialización:}

Fase de la actividad pesquera que comprende el consumo en la región, la comercialización en el mercado nacional y en el externo.

La comercialización al estado fresco/refrigerado es libre; el número de comerciantes mayoristas y minoristas es de 250 aproximadamente; la producción de las empresas es comercializada en el mercado nacional y externo por ellos mismos.

\subsection{Capacitación:}

Dentro de las limitaciones de los pescadores artesanales se tiene: la falta de formación profesional, de programas de capacitación permanentes y transferencia tecnológica, lo cual no permite al pescador artesanal tener mejores perspectivas para realizar las faenas de pesca y elevar su nivel socioeconómico.

En los últimos años el ex Ministerio de Pesqueria a través de la Dirección Nacional de Pesca Artesanal, del Instituto Tecnológico Pesquero, del Centro de Entrenamiento Pesquero Paita y PADESPA viene promoviendo en forma permanente cursos de capacitación relacionados con la actividad extractiva, cultivos, de procesamiento, manipulación, preservación, reparación y mantenimiento de motores, etc.

\section{IMPACTO AMBIENTAL DE LAS ACTIVIDADES ACUÍCOLAS EN LA REGIÓN TACNA}

La actividad acuicola continental en el sur del Perú, al igual que en la mayor parte del pais, se sustenta básicamente en la crianza extensiva, semiintensiva e intensiva de una especie propia de aguas frías, como es la trucha arco iris Oncorhynchus mykiss, utilizándose, por consiguiente, los recursos hídricos (rios y lagunas) localizados en la zona altoandina para la 
práctica de esta actividad, que cada día está cobrando gran importancia.

La actividad acuícola marina en la región Tacna está orientada básicamente al cultivo extensivo (poblamiento y repoblamiento) del recurso "chanque" o "tolina" Concholepas concholepas, el mismo que viene siendo ejecutado por las asociaciones de pescadores artesanales, que cuentan con la respectiva autorización de la Dirección Regional de la Producción de Tacna.

\subsection{En el Medio Ambiente:}

Teniendo presente la premisa fundamental de que la crianza de trucha en forma extensiva en una laguna, no va a causar mayormente un impacto negativo sobre las condiciones bioecológicas de ese ambiente acuático, vamos a tratar de identificar algunos posibles efectos que se pueden presentar en el medio ambiente, luego de la puesta en marcha de la actividad proyectada, en el mediano y largo plazo.

\section{a) Sobre la Flora y Fauna}

El impacto de la introducción y subsiguiente desarrollo de una población de trucha sobre la flora acuática se podría manifestar en una primera instancia en un posible incremento en la densidad de las poblaciones de microalgas, debido fundamentalmente a la disminución de los consumidores de primer orden (zooplancton, perifiton y algunos representantes del bentos) por acción de la trucha al alimentarse de éstos. Es decir, que al disminuir la cantidad de estos consumidores, las microalgas o fitoplancton van a ser depredadas en menor magnitud, existiendo por consiguiente, la posibilidad de un mayor crecimiento poblacional. El efecto directo sobre las macrofitas acuáticas (vegetales macroscópicos) no va a ser significativo, por cuanto la trucha, como es bien conocido, no se alimenta de ellas.

El impacto más significativo será sobre las poblaciones de animales invertebrados, que sirven de alimento a la trucha, tales como los representantes del zooplancton (microcrustáceos: ciclopoideos, calanoideos, cladóceros, ostrácodos, etc.), perifiton (anfipodos: gammarus y hyalella y larvas de insectos de las órdenes diptera, plecoptera, odonata, etc.) y bentos (anélidos, platelmintos, etc.). Es muy posible que las poblaciones de todos estos organismos se vean disminuidas por efecto de la depredación ejercida por la trucha introducida en la laguna.

Es muy remota la idea de pensar que la producción de materia orgánica, conformada por sustancias excrementicias de las poblaciones de trucha introducidas anualmente en la laguna, así como también restos vegetales y animales, va a ser enorme, hasta tal punto que su acumulación vaya originar algún problema de eutroficación. Una de las razones para asegurar que es prácticamente imposible que ocurra este fenómeno de degradación del ambiente acuático, es la buena oxigenación que presentan las aguas de la lagunas de la región, lo cual se debe a la elevada tasa de fotosíntesis que realizan las microalgas y fanerógamas acuáticas presentes, coadyuvada con la tasa de recambio de agua y movimientos (olas y corrientes); ya que es conocido, por principios ecológicos elementales, que al existir oxígeno en cantidades suficientes toda la materia orgánica que pueda producirse será inmediatamente oxidada y gracias a los microorganismos descomponedores será transformada a nutrientes inorgánicos. Es decir que el agua seguirá siendo de buena calidad, no solamente para fines acuícolas, sino también para otros, tales como consumo animal y humano.

\section{b) Sobre ecosistemas acuáticos y terrestres}

Inicialmente es posible que se altere, aunque levemente, el equilibrio ecológico en el ecosistema acuático, por las razones mencionadas en el punto anterior; pero, como todo ambiente dinámico, es muy posible también que dicho ecosistema tienda a estabilizarse y logre un nuevo equilibrio bioecológico, en un plazo relativamente corto.

Sobre los ecosistemas terrestres adyacentes al ambiente acuático utilizado, la actividad proyectada no tendrá mayormente ningún tipo de efecto o impacto.

\section{c) Sobre el paisaje}

La actividad de crianza de trucha puede tener un efecto positivo, en el sentido de mejorar las condiciones ambientales del paisaje en la laguna y en su entorno. Este mejoramiento puede ser utilizado para propiciar y/o programar acciones de turismo hacia la zona.

\subsection{En la Socio-economía:}

La población económicamente activa (PEA) a nivel de la provincia de Candarave se estima que es alrededor del $35 \%$ de la población total. Si consideramos que esa población total, según el último censo de 1993, es de 8292 personas, resulta entonces que la PEA estará compuesta aproximadamente por 2902 personas.

Quiere decir entonces que un poco más de la tercera parte de la población mantiene a las otras tres cuartas partes de la población total. Además, es importante considerar que debido a la movilización social que se produce de las 
localidades altoandinas y del campo hacia la capital de la región, origina que la PEA se concentre en dicho centro. Considerando todos estos aspectos, surge la necesidad, entre otras cosas, de dotar de mayores fuentes alimenticias (caso de los productos hidrobiológicos) y crear nuevas fuentes de trabajo a esa población campesina, que está evidentemente arraigada en las labores agrícolas

La crianza de trucha, que es una de las actividades acuicolas continentales más importantes en el ámbito regional, va a tener realmente un impacto bastante favorable sobre los principales aspectos socioeconómicos de los pobladores del área de influencia, manifestado de la siguiente manera:

- Va a generar nuevos puestos de trabajo.

- Posibilidad de mejorar sus ingresos económicos.

- Diversificación de sus actividades tradicionales, con la inclusión de una nueva como es la piscicultura.

- Posibilidad de mejorar su dieta alimenticia, con la inclusión de proteina animal de origen íctico.

- Al mejorar sus ingresos económicos, existe la posibilidad de elevar el status socio-económico de esos pobladores.

También se genera la captación de mano de obra, la misma que puede ser en forma directa e indirecta. La primera se hará a través de la ocupación en las tareas propias de la actividad piscicola que se va a desarrollar. Se estima que se dará trabajo en forma casi permanente a por lo menos diez (10) personas, las cuales cumplirán las siguientes tareas: adquisición y traslado de los alevinos, siembra, vigilancia, controles biométricos, cosecha, selección, limpieza, acondicionamiento, etc.

La mano de obra indirecta se captará a través de la generación de actividades conexas a la producción de trucha, tales como el comercio, transporte y posiblemente también en actividades de transformación (conservas, ahumado, etc.).

\subsection{Sobre la Comunidad:}

Como se ha mencionado anteriormente, el impacto que va a tener la actividad acuícola continental sobre las comunidades campesinas ubicadas en la zona altoandina de la región, no podrá ser otro que de carácter favorable. Esto se va a manifestar a través de la oferta de una fuente adicional de trabajo, posibilidad de adquisición de otro ingrediente de su dieta alimenticia (carne de trucha), etc. Es importante mencionar que, según dispositivos legales, existe la posibilidad de que las comunidades aledañas puedan realizar la extracción de trucha de un ambiente acuático otorgado en concesión para la práctica de la acuicultura, empleando caña de pescar. La trucha obtenida bajo este sistema se entiende que será destinada exclusivamente para el autoconsumo.
Las comunidades beneficiadas con la ejecución de actividades acuícolas en la región Tacna serán las siguientes: Huaytire, Aricota, Curibaya, Huanuara, Quilahuani, Pallata, Candarave, San Pedro, Totora, Chintari, Mirave, Ilabaya, etc.

\section{EVALUACIÓN DE IMPACTO AMBIENTAL DE L A A C T I V I D A D DE PROCESAMIENTO PESQUERO}

Los efectos directos favorables de la puesta en marcha de una instalación pesquera, en los aspectos socioecómicos de la región serán los siguientes:

- Generación de mayor valor agregado, aumentando la producción y contribuyendo a la reactivación económica de la región y el pais, lo que redundará en la superación de la recesión que ha venido adoleciendo nuestra economía.

- Aumento del ingreso de divisas al país, ya que la mayor parte de la producción industrial pesquera está destinada al mercado exterior.

- Desarrollo tecnológico al modernizar las maquinarias y equipos, mejorar los procedimientos técnicos, elevar la productividad, a fin de obtener mejores precios y reducir costos.

- Estimulo a la inversión, al ser una muestra de estabilidad para los inversionistas privados, quienes están llamados a desempeñar el rol fundamental en la producción, de acuerdo con la política económica vigente en el país y en la economía internacional.

- Creación de nuevos puestos de trabajo y empleo, lo que implica coadyuvar a la reducción de la alta tasa de desempleo observada en la región sur del país, lo que consiguientemente propiciará la generación de ingresos para los trabajadores y sus familias, mejorando el nivel de vida de los mismos.

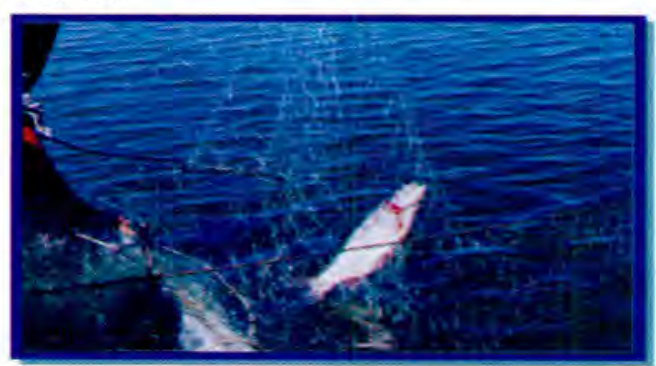

Extracción de trucha, principal actividad acuicola continental en Tacna.

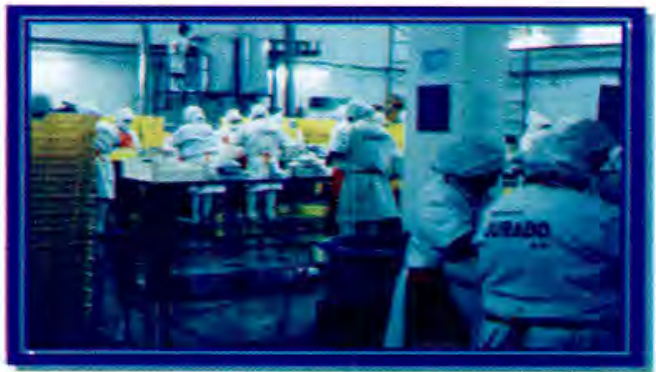

Procesamiento dé recursos hidrobiológicos, principal actividad industrial pesquera en Tacna. 